\title{
Qual o papel da aspirina na prevenção primária do carcinoma colorretal em indivíduos de médio risco? Uma revisão baseada na evidência
}

Sílvia Colmonero Martins, ${ }^{1}$ Sofia Cardoso, ${ }^{2}$ Ana Raquel Marques ${ }^{3}$

\section{RESUMO}

Introdução: O carcinoma colorretal (CCR) é a $2^{\text {a }}$ causa de mortalidade por neoplasia nos países desenvolvidos. A prevenção primária assenta em mudanças de estilo de vida, estando em investigação a «quimioprevenção». Alguns estudos têm demonstrado benefício da Aspirina ${ }^{\circledR}$ (AAS) na prevenção do CCR.

Objetivo: Determinar a evidência do efeito da AAS na incidência e mortalidade por CCR em indivíduos adultos de médio risco. Métodos: Foram identificados ensaios controlados aleatorizados (ECA), meta-análises (MA), revisões sistemáticas (RS), guidelines baseadas na evidência, assim como revistas portuguesas de cuidados de saúde primários e bases de dados eletrónicas, incluindo MEDLINE e Cochrane Library. A pesquisa foi limitada a publicações realizadas entre 1/4/2006 e 1/4/2016, em língua portuguesa, inglesa e espanhola, utilizando os termos MESH: Aspirin, Primary prevention e Colorectal neoplasms. Para atribuição de força de recomendação (FR) e de níveis de evidência foi utilizada a escala Strengh of Recomendation Taxonomy (SORT), da American Family Physician.

Resultados: Obtiveram-se 75 artigos, dos quais sete cumpriam os critérios de inclusão: uma NOC, uma MA, três RS, um estudo de coorte e um estudo original. A NOC não recomenda a AAS para prevenir o CCR em indivíduos de risco médio de CCR com FR (SORT B). Globalmente, os estudos parecem mostrar que a AAS, em dose $\geq 300 \mathrm{mg} /$ dia, administrada durante $\geq 5$ anos poderá reduzir a incidência de CCR com efeito mais pronunciado 10 anos após o início da toma. Doses inferiores, administradas com menor frequência e com duração de tratamento inferior, parecem ser menos efetivas.

Conclusões: Conclui-se que a AAS, administrada de forma regular, poderá ser efetiva na redução da incidência do CCR, com eventual impacto na mortalidade por CCR e com uma FR B (SORT B). Contudo, o seu uso deve ser recomendado com cautela pela incidência aumentada de efeitos adversos, como a hemorragia gastrointestinal. ECAs futuros são necessários para esclarecer a dose e duração que permita ao indivíduo ter um benefício oncológico máximo e um mínimo risco hemorrágico.

\section{INTRODUÇÃO}

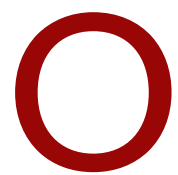

O carcinoma colorretal (CCR) é a segunda neoplasia mais comum e a segunda causa de morte por neoplasia nos países desenvolvidos, com risco de $5 \%$ ao longo da vida e cerca de um milhão de novos casos em todo mundo por ano. ${ }^{1-3}$

1. USF Lagoa - ULS Matosinhos. ORCID nº 0000-0002-1600-8571

2. USF Maresia - ULS Matosinhos.

3. UCSP São Mamede Infesta - ULS Matosinhos.
Em 2012, na Europa, registou-se uma taxa de incidência de CCR de $43,5 \%$ e de mortalidade de $19,5 \%$, com dados similares em Portugal (incidência 42,3\%; mortalidade $14,5 \%) .^{1-2}$ Isto demonstra que as estratégias preventivas devem ser melhoradas.

A maioria dos CCR desenvolve-se a partir de lesões pré-malignas na sua história natural (sequência adenoma-carcinoma).$^{3-4}$ O CCR apresenta um período longo e silencioso de estádio pré-maligno no qual surgem lesões percursoras de CCR (adenomas) ou CCR precoce assintomático. O rastreio é essencial, especialmen- 
te após os 50 anos (idade a partir da qual ocorrem $90 \%$ dos casos), permitindo a deteção e tratamento precoces, ainda numa fase de cura. ${ }^{2,4}$

A prevenção primária assenta em mudanças de estilo de vida, estando há vários anos em investigação a «quimioprevenção», enquanto potencial complemento ao rastreio.

Estudos experimentais têm investigado o papel da aspirina (AAS) na prevenção primária do CCR, à semelhança do seu papel na prevenção cardiovascular (CV). Alguns estudos têm demonstrado benefício da AAS na prevenção de neoplasias, incluindo o CCR. Contudo, está ainda por esclarecer se o potencial benefício cardiovascular e oncológico transpõe o risco hemorrágico associado à administração deste fármaco. ${ }^{2,5}$

Pretende-se, com esta revisão, determinar a evidência do efeito da AAS na incidência do CCR e na mortalidade por CCR, em indivíduos adultos de médio risco.

\section{MÉTODOS}

Em abril de 2016 foi realizada uma pesquisa de normas de orientação clínica (NOC), meta-análises (MA), revisões sistemáticas (RS) e ensaios clínicos aleatorizados (ECA) na MEDLINE, em sites médicos baseados em evidência (The Cochrane Library;DARE; Bandolier; Guidelines Finder, da National Eletronic Library for Health, no NHS britânico; National Guideline Clearinghouse; Canadian Medical Association Practice Guidelines InfoBase) e no Índex de Revistas Médicas Portuguesas, publicados entre 1 de abril de 2006 e 1 de abril de 2016, em língua portuguesa, inglesa e espanhola, utilizando os seguintes termos MESH: Aspirin; Primary preventione Colorectal neoplasms. Foram ainda usados os termos portugueses: "Aspirina", "Prevenção primária" e "Neoplasias intestinais".

Foram incluídos estudos realizados na população adulta (idade $\geq 18$ anos) de risco médio para CCR, na qual se integram indivíduos sem fatores de risco conhecidos para adenomas colorretais (ACR) ou CCR, assim como indivíduos com história pessoal ou familiar de ACR e familiar de CCR esporádico (população). Os estudos incluídos deveriam ter um grupo exposto à terapêutica com AAS (intervenção), comparativamente a um grupo placebo/ausência de tratamento/associação de AAS com técnicas de prevenção secundária de CCR (i.e., colonoscopia/sigmoidoscopia). O resultado medido (outcome primário) foi o efeito da AAS na inci- dência e mortalidade por CCR. Quando possível, foram também medidos os efeitos colaterais potenciais mais comuns da AAS, nomeadamente a hemorragia gastrointestinal (HGI) e o acidente vascular cerebral (AVC) (outcomes secundários).

Foram excluídos estudos realizados em indivíduos com idade < 18 anos; em doentes portadores de doença cardiovascular estabelecida; com antecedentes pessoais atuais ou passados de CCR; ou com risco aumentado de hemorragia. Foram também excluídos artigos de opinião, editoriais, letters e notícias, e estudos não aleatorizados ou realizados em animais. Estudos duplicados ou já incluídos nas RS ou MA selecionadas foram também excluídos.

A seleção dos trabalhos pelo título e resumo foi efetuada por todas as autoras. Os artigos selecionados para leitura integral foram lidos por, pelo menos, duas das autoras para decidir a sua inclusão. Após leitura integral, constatou-se que uma MA incluía apenas uma RS cujo outcome era o preconizado por esta revisão, a qual foi encontrada por backward citation e integrada na análise desta revisão, com exclusão da MA original.

A avaliação final da qualidade e nível de evidência dos artigos incluídos foi discutida e decidida por consenso entre todas as autoras. O nível de evidência (NE) e a força de recomendação (FR) dos artigos, quando ausentes nos artigos originais, foram atribuídos pelas autoras, de acordo com os critérios da escala Strength of Recommendation Taxonomy (SORT), da American Family Physician. ${ }^{6}$

\section{RESULTADOS}

Utilizando os termos de pesquisa nas bases de dados previamente indicadas foram encontrados 75 artigos, dos quais foram excluídos 22 por estarem duplicados. As restantes publicações foram revistas por leitura dos títulos, resumos e texto integral, tendo sido excluídos 46 artigos no total porque o tema e objetivos eram díspares do pretendido, e/ou por não estarem de acordo com os critérios de inclusão definidos. O fluxograma de seleção dos artigos está representado na Figura 1.

No final obtiveram-se sete artigos integrados e avaliados pelas autoras nesta revisão: uma NOC; uma MA; três RS; um estudo de coorte; e um estudo original. $\mathrm{O}$ resumo do corpo de evidência encontra-se explanado nos Quadros I a VII. 




Figura 1. Fluxograma de seleção dos artigos.

\section{Norma de orientação clínica (NOC)}

A NOC da U.S. Preventive Services Task Force (USPSTF), de 2007, recomenda que a AAS não seja administrada para prevenir o CCR em indivíduos assintomáticos de médio risco para CCR - Força de Recomendação D (escala USPSTF) equiparada à Força de Recomendação B (escala SORT) (Quadro I). A USPSTF demonstrou boa evidência de que a AAS aumenta o risco de HGI e evidência limitada de que aumenta o risco de AVC hemorrágico.

\section{Meta-análise (MA)}

A MA de Enrico Flossmann e colaboradores (2007) ${ }^{4}$
(Quadro II) incluiu dois ECAs (UK-TIA trial; e o British Doctors Aspirin Trial) que mostraram redução significativa na incidência de CCR $(\mathrm{HR}=$ 0,26; IC95\% [0,12$0,56, p=0,0002]) \mathrm{com}$ a utilização da AAS durante cinco ou mais anos, na dose de $300-1.200 \mathrm{mg} / \mathrm{dia}$, com uma adesão à terapêutica adequada pelos indivíduos e após um follow-up de 10 a 14 anos desde o início da toma de AAS. Não ocorreu efeito significativo na incidência de CCR com dose $>1.200 \mathrm{mg} /$ dia e follow-up de 15 ou mais anos.

Os autores desta MA fizeram também uma revisão sistemática (RS) de estudos observacionais (Quadro II). As doses e duração de tratamento com AAS foram variáveis. Os estudos caso-controlo (CC) mostraram redução do risco de CCR (OR=0,80; IC95\% [0,73-0,87, $p<0,0001]$ ), mais significativa quando a AAS era administrada na dose de $300 \mathrm{mg} /$ dia ou superior, durante 10 ou mais anos (OR=0,64; IC95\% [0,59-0,70, $p<0,0001])$. Os estudos de coorte (EC) mostraram o mesmo efeito com doses de $300 \mathrm{mg} /$ dia ou superiores, durante 10 a 20 anos,

\begin{tabular}{|c|c|c|c|}
\hline Autor, Ano & Conclusão & $\mathrm{NE}^{*}$ & FR \\
\hline $\begin{array}{l}\text { Routine aspirin or nonsteroidal } \\
\text { anti-inflammatory drugs for } \\
\text { the primary prevention of } \\
\text { colorectal cancer: U.S. } \\
\text { Preventive Services Task Force } \\
\text { - USPSTF Recommendation } \\
\text { Statement (2007) }{ }^{1}\end{array}$ & $\begin{array}{l}\text { - A AAS em baixa dose ( } 100-325 \mathrm{mg} \text { ) não } \\
\text { reduz a incidência de CCR. } \\
\text { - A AAS em alta dose (> } 14 \text { comprimidos/ } \\
\text { semana de } 325 \mathrm{mg} \text { ) e por períodos } \\
\text { prolongados de tempo (> } 10 \text { anos) pode } \\
\text { reduzir a incidência de CCR. } \\
\text { - A AAS diminui a mortalidade por CCR. } \\
\text { - A AAS aumenta o risco de HGI. } \\
\text { - A AAS aumenta o risco de AVC hemorrágico. }\end{array}$ & $\begin{array}{l} \\
\text { NE } 3 \\
\text { NE } 1 \\
\text { NE } 2\end{array}$ & $\begin{array}{l}\text { Não recomenda a administração de } \\
\text { AAS para prevenir o CCR em } \\
\text { indivíduos de médio risco para CCR. }\end{array}$ \\
\hline
\end{tabular}

Legenda: AAS = aspirina; $\mathrm{AVC}=$ acidente vascular cerebral; $\mathrm{CCR}=$ carcinoma colorretal; $\mathrm{FR}$ = força de recomendação; $\mathrm{HGI}=$ hemorragia gastrointestinal; NE* = nível de evidência, atribuído pela escala SORT; SORT = Strength of Recommendation Taxonomy. 


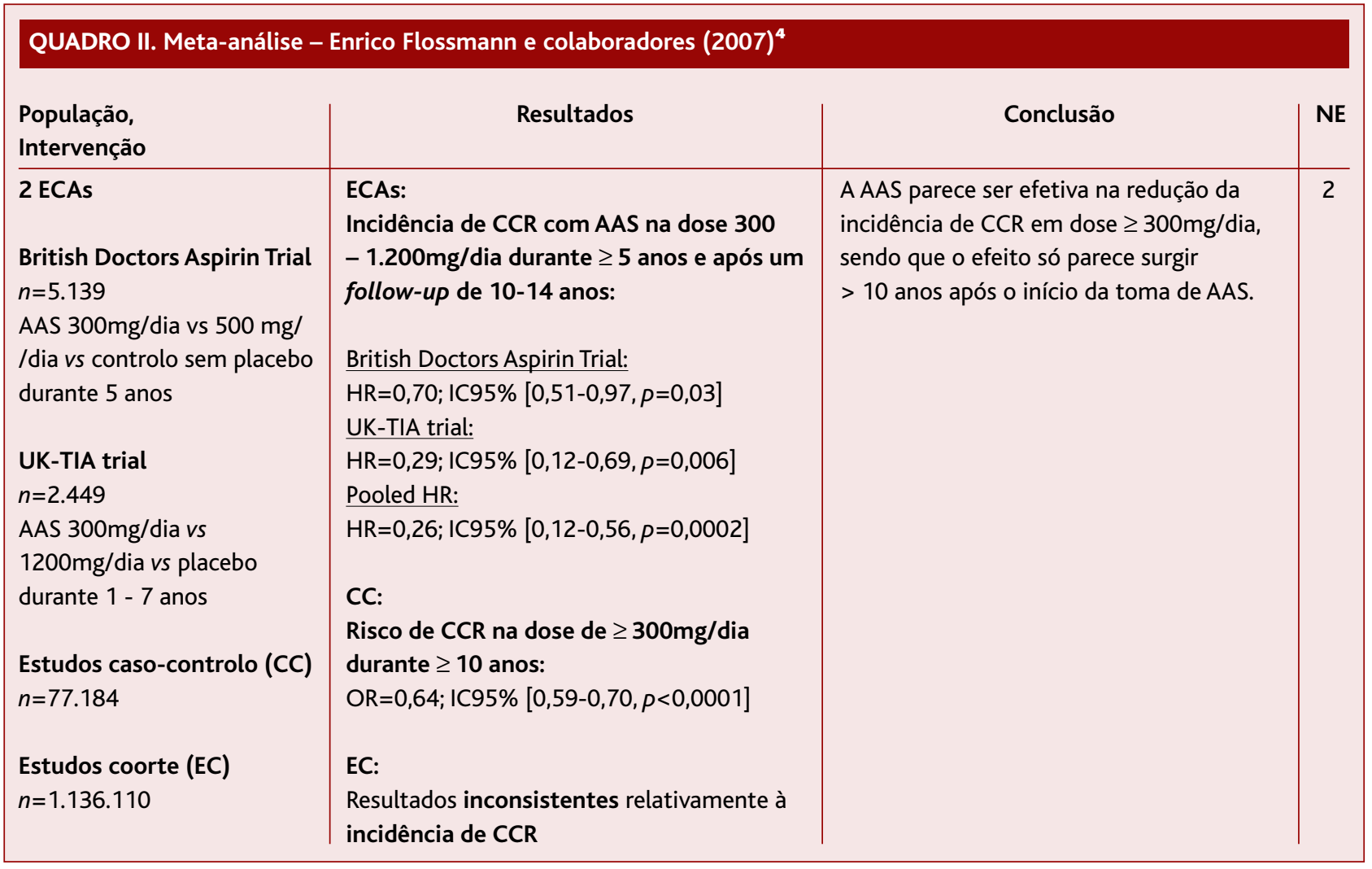

Legenda: $\mathrm{AAS}=$ aspirina; $\mathrm{CCR}=$ cancro colorretal; $\mathrm{CC}=$ estudo caso-controlo; $\mathrm{EC}=$ estudo de coorte; $\mathrm{ECA}=$ ensaio clínico aleatorizado; $\mathrm{NE}=$ nível de evidência.

mas com grande heterogeneidade nas doses e duração, o que não permitiu fazer uma análise agrupada.

Os dois ECAs e estudos observacionais incluídos nesta MA apresentaram tamanho adequado, clareza quanto à distribuição por grupos, resultados consistentes e intenção de tratar. O ECA (UK-TIA trial) apresentou ocultação e follow-up adequados (ensaio duplamente cego; mais de $80 \%$ dos participantes mantiveram a toma de AAS durante o período de estudo), enquanto o ECA (British Doctors Aspirin Trial) era «não cego» e com follow-up inferior a $80 \%$, pelo que se atribuiu um NE 2 a esta MA.

\section{Revisões sistemáticas (RS)}

\subsection{Peter Rothwell e colaboradores (2010)}

Uma meta-análise de Stucliffe e colaboradores (de 2013) ${ }^{8}$ apenas incluiu uma RS (Rothwell e colaboradores), ${ }^{3}$ de acordo com o outcome preconizado por esta re- visão, encontrada por backward citation (Quadro III).

Trata-se de uma RS que integrou quatro ECAs. Demonstrou que a AAS administrada durante cinco ou mais anos, numa dose de $75 \mathrm{mg} /$ dia ou superior, reduz a incidência (HR=0,35; IC95\% $[0,20-0,63, p<0,0001]) \mathrm{e}$ mortalidade (HR=0,24; IC95\% [0,11-0,52, $p<0,0001]$ ) por carcinoma do cólon após 20 anos de follow-up.

Esta redução foi particularmente significativa para o carcinoma do cólon proximal (incidência: $\mathrm{HR}=0,45$; IC95\% [0,28-0,74, $p=0,001]$; mortalidade: $\mathrm{HR}=0,34$; IC95\% $[0,18-0,66, p=0,001])$.

Registou-se também uma redução significativa da incidência (HR=0,58; IC95\% [0,36-0,92, $p=0,02]$ ) e mortalidade (HR=0,47; IC95\% $[0,26-0,87, p=0,01])$ por carcinoma retal quando a AAS foi administrada por cinco ou mais anos.

Não houve redução significativa na incidência e mortalidade por carcinoma do cólon distal, pelo que não foram extraídas conclusões. 


\begin{tabular}{|c|c|c|c|}
\hline $\begin{array}{l}\text { População, } \\
\text { Intervenção }\end{array}$ & Resultados & Conclusão & NE \\
\hline $\begin{array}{l}4 \text { ECAs } \\
n=14.033 \\
\text { AAS } \geq 75 \mathrm{mg} / \text { dia } \\
\text { vs } \\
\text { placebo durante } \geq 5 \text { anos }\end{array}$ & $\begin{array}{l}\text { Após } 20 \text { anos de follow- } u p \text { : } \\
\text { Incidência de carcinoma do cólon: } \\
\text { HR=0,35; IC95\% }[0,20-0,63, p<0,0001] \\
\text { Mortalidade por carcinoma do cólon: } \\
\text { HR=0,24; IC95\% [0,11-0,52, } p<0,0001] \\
\text { Incidência de carcinoma do cólon proximal: } \\
\text { HR=0,45; IC95\% [0,28-0,74, } p=0,001] \\
\text { Mortalidade por carcinoma do cólon proximal: } \\
\text { HR=0,34; IC95\% [0,18-0,66, } p=0,001] \\
\text { Incidência de carcinoma retal: } \\
\text { HR=0,58; IC95\% [0,36-0,92, } p=0,02] \\
\text { Mortalidade por carcinoma retal: } \\
\text { HR=0,47; IC95\% }[0,26-0,87, p=0,01] \\
\text { Incidência de carcinoma do cólon distal: } \\
\text { HR=1,10; IC95\% }[0,73-1,64, p=0,66] \\
\text { Mortalidade por carcinoma do cólon distal: } \\
\text { HR=1,21; IC95\% }[0,66-2,24, p=0,54]\end{array}$ & $\begin{array}{l}\text { A AAS reduziu a incidência e } \\
\text { mortalidade por carcinoma do cólon. } \\
\text { A redução foi particularmente } \\
\text { significativa para o carcinoma do cólon } \\
\text { proximal. }\end{array}$ & 1 \\
\hline
\end{tabular}

Legenda: $A A S=$ aspirina; $E C A$ = ensaio clínico aleatorizado; $N E$ = nível de evidência.

A RS de Rothwell e colaboradores incluiu ECAs de elevada qualidade metodológica, foi clara quanto à distribuição por grupos, ocultação, intenção de tratar, bem como apresentou tamanho da população e follow-up adequados, pelo que se atribuiu um NE 1.

\subsection{Catherine Dube e colaboradores (2007)}

A RS de Catherine Dube e colaboradores ${ }^{9}$ incluiu quatro ECAs (Quadro IV), mas apenas dois ECAs (Physicians' Health Study; e Women's Health Study), de "boa qualidade" pela classificação da USPSTF, avaliaram os outcomes desta revisão, mostrando não existir redução significativa na incidência de CCR (RR=1,02; IC95\% $[0,84-1,25])$ quando a AAS foi usada na dose de 100 a $325 \mathrm{mg} /$ dia durante cinco a dez anos. Nenhum ECA avaliou a mortalidade por CCR.

Esta RS incluiu também EC, de qualidade «fraca» a «boa», relativamente aos outcomes preconizados pelas autoras. No EC Cancer Prevention Study II houve redução significativa na mortalidade por CCR em homens
$(\mathrm{RR}=0,58$; IC95\% [0,36-0,93]) e mulheres $(\mathrm{RR}=0,61$; IC95\% [0,38-0,97]) com a utilização de AAS por 15 ou mais anos, em doses variáveis (100-325mg/dia). Oito EC mostraram redução significativa na incidência de CCR (RR=0,78; IC95\% $[0,63-0,97])$, quando a AAS era administrada durante quatro a 12 anos, mas não especificaram a dose mínima para obtenção deste efeito.

Esta RS incluiu ainda estudos CC, a maioria de qualidade «limitada». Sete mostraram resultados inconsistentes relativamente à incidência de CCR, sendo que apenas três favoreceram a redução da incidência deste carcinoma, com a AAS em doses e duração variável, impossibilitando a análise agrupada.

Esta RS avaliou os efeitos adversos hemorrágicos da AAS. Uma RS mostrou aumento do risco de AVC hemorrágico. Outras RS mostraram risco aumentado de HGI. Estes efeitos adversos são dependentes da dose e duração do tratamento, mas os estudos não são consensuais na dose a partir do qual estes ocorrem, havendo relatos de ocorrência para doses $\geq 100 \mathrm{mg} /$ dia. 


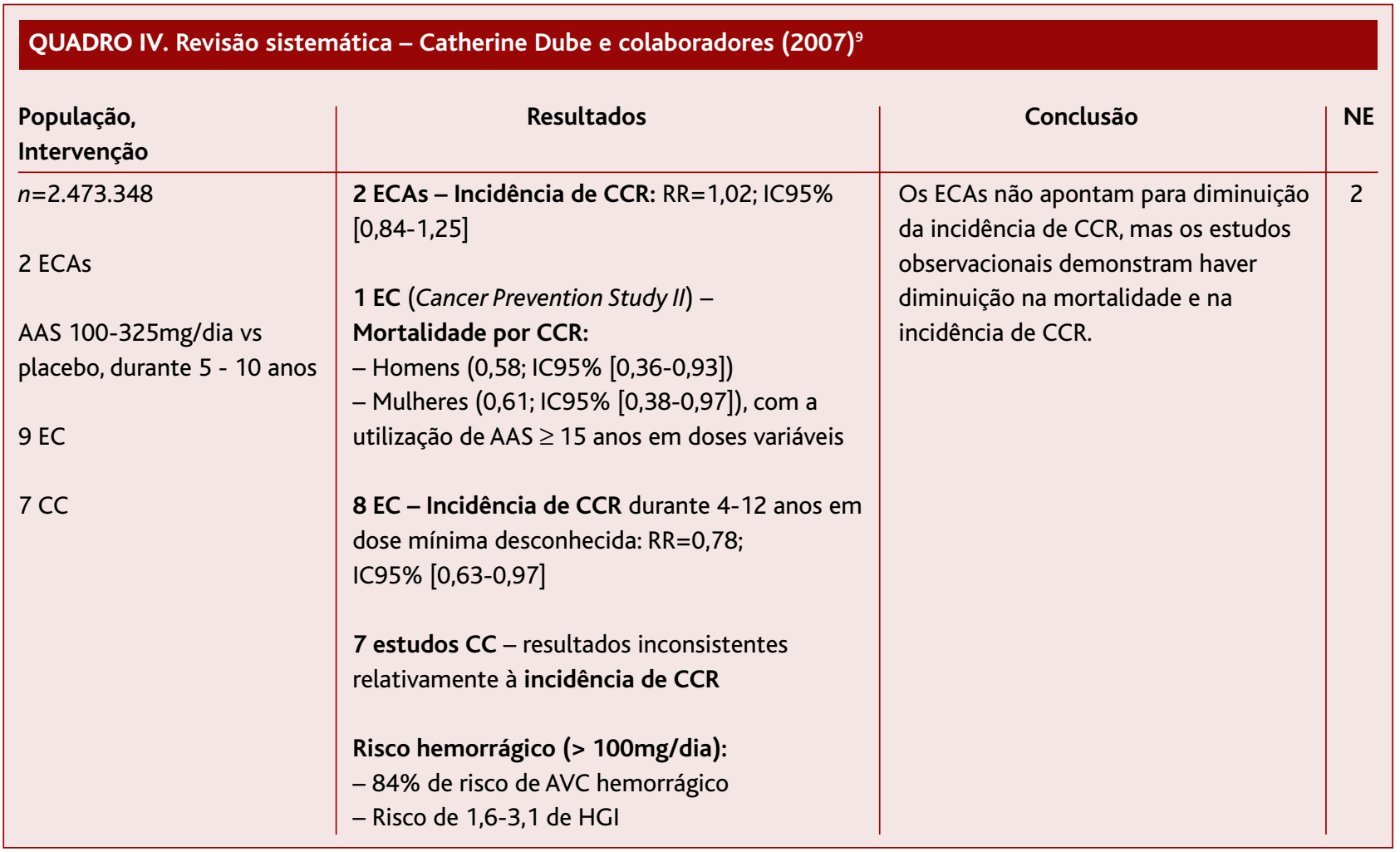

Legenda: $\mathrm{AAS}=$ aspirina; $\mathrm{AVC}=$ acidente vascular cerebral; $\mathrm{CCR}=$ carcinoma colorretal; $\mathrm{CC}=$ estudos caso-controlo; $\mathrm{EC}=$ estudos de coorte; $\mathrm{ECA}=$ ensaio clínico aleatorizado; $\mathrm{HGl}=$ hemorragia gastrointestinal.

Assim, a RS de Catherine Dube e colaboradores analisou ECAs de «boa qualidade» e estudos observacionais de «fraca a boa qualidade». Os ECAs não apontam para diminuição da incidência de CCR, mas os estudos observacionais demonstram haver diminuição na mortalidade e da incidência de CCR, que parece ser mais significativa com doses e duração superior do tratamento. A heterogeneidade das doses de AAS administrada e as diferenças no tempo de intervenção, bem como a existência de resultados inconsistentes, levanos a atribuir um NE 2 a esta RS.

\subsection{Alaa Rostom e colaboradores (2007) ${ }^{2}$}

A RS de Alaa Rostom e colaboradores ${ }^{2}$ englobou 39 estudos (Quadro V): ECAs, EC e CC.

Apenas um ECA avaliou a incidência de CCR com AAS em dose de 81 a 325mg/dia e, mesmo após 12 anos de tratamento, não mostrou redução desse outcome (RR=1,03; IC95\% [0,83-1,28]).

Os EC mostraram que o uso regular de AAS reduz a incidência de CCR (RR=0,78; IC95\% [0,63-0,97]) com redução do risco relativo de 15 a 40\%. Um EC mostrou que a AAS, administrada por 20 ou mais anos, reduzia a incidência de CCR (RR=0,56; IC95\% [0,36-0,9]). Outro EC mostrou o mesmo efeito quando administrada mais de duas vezes por semana por um período superior a dois anos (RR=0,54; IC95\% [0,34-0,83]). Dois EC mostraram efeito inconsistente da AAS na redução da mortalidade por CCR: um favoreceu o uso (integrado na RS de Catherine Dube e colaboradores $)^{9} \mathrm{e}$ outro não mostrou redução da mortalidade por CCR $(\mathrm{SMR}=1,05$; IC95\% $[0,9-1,2])$.

Os estudos CC apresentaram grande heterogeneidade na exposição à AAS, dificultando a análise agrupada dos dados. Estes mostraram que a AAS administrada por quatro a seis anos poderia reduzir a incidência de CCR (RR=0,68; IC95\% [0,54-0,8]), mas sem efeito se o tratamento tiver uma duração inferior a quatro anos. Dois estudos CC avaliaram a dose de AAS capaz de reduzir a incidência de CCR; apenas a dose de 


\begin{tabular}{|c|c|c|c|}
\hline $\begin{array}{l}\text { População, } \\
\text { Intervenção }\end{array}$ & Resultados & Conclusão & NE \\
\hline $\begin{array}{l}n=662.424 \\
39 \text { estudos: } \\
\text { ECAs } \\
\text { EC } \\
\text { CC } \\
\text { AAS vs placebo }\end{array}$ & $\begin{array}{l}1 \text { ECA (Physicians Health Study) - incidência de } \\
\text { CCR (AAS 81-325mg/dia vs placebo, durante } \\
>12 \text { anos): RR=1,03; IC95\% [0,83-1,28] } \\
\text { EC - Incidência de CCR com uso regular de } \\
\text { AAS: RR=0,78; IC95\% }[0,63-0,97] \\
\text { CC - Incidência de CCR com uso de AAS por } \\
\text { 4-6 anos: RR=0,68; IC95\% [0,54-0,8] } \\
\text { Risco hemorrágico (> 100mg/dia): } \\
\text { - RR HGI 1,5-3,0 } \\
\text { - } 84 \% \text { de risco de AVC hemorrágico }\end{array}$ & $\begin{array}{l}\text { A AAS parece reduzir a incidência de } \\
\text { CCR, mas apenas os estudos } \\
\text { observacionais favorecem a utilização } \\
\text { da AAS com redução do risco relativo } \\
\text { na incidência de CCR de } 15-40 \% \text {. }\end{array}$ & 2 \\
\hline
\end{tabular}

Legenda: $\mathrm{AAS}=$ aspirina; $\mathrm{AVC}=$ acidente vascular cerebral $\mathrm{CCR}=$ carcinoma colorretal; $\mathrm{CC}=$ estudos caso-controlo; $\mathrm{EC}=$ estudos de coorte; $\mathrm{ECA}=$ ensaio clínico aleatorizado; $\mathrm{HGl}=$ hemorragia gastrointestinal.

$350 \mathrm{mg} /$ dia (RR=0,60; IC95\% [0,5-0,9]), num dos estudos, e de $300 \mathrm{mg} / \mathrm{dia}$ (RR=0,60; IC95\% [0,4-0,9]), no outro estudo, tiveram esse efeito.

Assim, esta RS demonstra que a AAS parece reduzir a incidência de CCR para doses e duração de tratamento progressivamente mais elevadas. Contudo, os resultados são inconsistentes, uma vez que apenas os estudos observacionais favorecem a utilização da AAS.

Os autores desta RS encontraram 11 RS que avaliaram os efeitos colaterais da AAS. A sua utilização, diariamente ao longo de vários meses, associou-se a risco de complicações gastrointestinais e de AVC hemorrágico. Os estudos apresentaram grande heterogeneidade nas doses suscetíveis de causar estes efeitos, os quais aumentam com doses progressivamente mais altas ( $\geq 100 \mathrm{mg} /$ dia).

Os estudos incluídos nesta RS apresentavam grande heterogeneidade nas doses e duração do tratamento com AAS, podendo interferir com o efeito do fármaco nos outcomes. A inconsistência entre os estudos observacionais e ECAs desta RS permitiu atribuir-lhe um NE 2.

\section{Estudo de coorte}

O coorte simulado de Cesare Hassan e colaborado- res (2012) ${ }^{10}$ (Quadro VI) envolveu uma população de 100.000 indivíduos integrados em ECAs, cujo outcome era o efeito da AAS na prevenção CV. Esses indivíduos, a quem era administrada AAS na dose de $75 \mathrm{mg} / \mathrm{dia}$, foram convidados a realizar o rastreio do CCR por colonoscopia total (CT) ou sigmoidoscopia (SC), pelo menos a cada 10 anos. O objetivo foi avaliar o efeito da AAS, isolada ou adicionada ao rastreio, na redução da mortalidade por CCR. Esses indivíduos tinham 50 anos de idade no início do estudo e foram acompanhados durante 30 anos. A AAS reduziu, por si só, em $41 \%$ a mortalidade por CCR, valor mais pronunciado quando adicionada à CT ou SC na prevenção do CCR.

O efeito benéfico do AAS foi contrabalançado pela ocorrência de 2.110 casos de HGI e 513 casos de AVC hemorrágico. A taxa de mortalidade relacionada com AAS ao longo da vida foi de $0,1 \%$ e a maioria dos participantes com efeitos colaterais tinham 65 anos ou mais. Trata-se de um coorte de elevada qualidade (prospetivo, com tamanho adequado e follow-up superior a $80 \%$ ), pelo que se atribuiu um NE 1.

\section{Estudo original}

O estudo original de Rob e colaboradores $(2015)^{5}$ (Quadro VII) surgiu no seguimento de mulheres com 


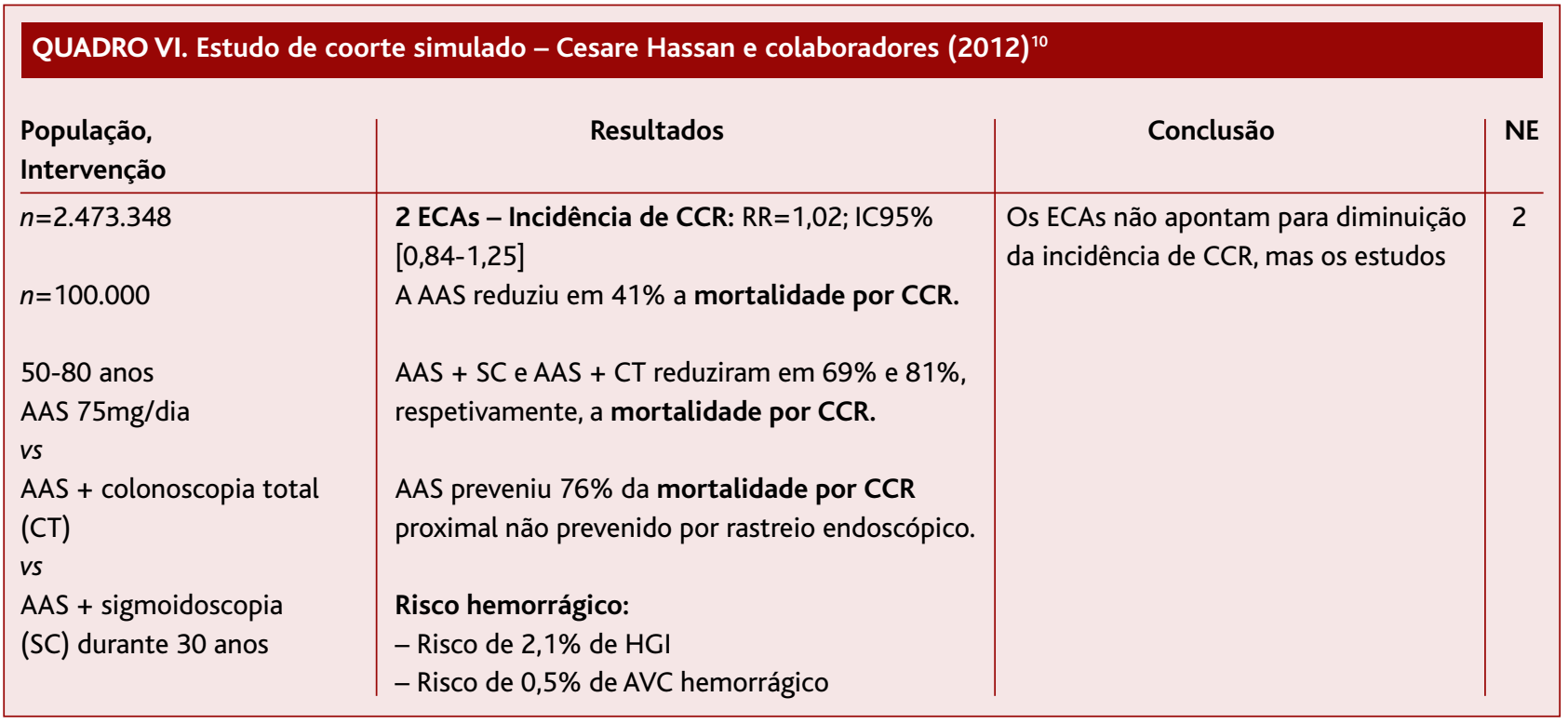

Legenda: $\mathrm{AAS}=$ aspirina; $\mathrm{AVC}=$ acidente vascular cerebral; $\mathrm{CCR}=$ carcinoma colorretal; $\mathrm{CC}=$ estudos caso-controlo; $\mathrm{EC}=$ estudos de coorte; $\mathrm{ECA}=$ ensaio clínico aleatorizado; $\mathrm{HGI}=$ hemorragia gastrointestinal.

\section{QUADRO VII. Estudo original - Rob Kruijsdijk e colaboradores $(2015)^{5}$}

\begin{tabular}{|c|c|c|c|}
\hline $\begin{array}{l}\text { População, } \\
\text { Intervenção }\end{array}$ & Resultados & Conclusão & NE \\
\hline $\begin{array}{l}n=27.939 \\
\text { AAS } 100 \mathrm{mg} \text { em dias } \\
\text { alternados } \\
\text { vs } \\
\text { placebo durante } 15 \text { anos }\end{array}$ & $\begin{array}{l}\text { Risco de CCR: } \\
\text { RRA=0,14\%; IC95\% [0,02-0,59\%] } \\
\text { Risco hemorrágico: } \\
\text { RRA=0,75\%; IC95\% [0,50-1,00\%] }\end{array}$ & $\begin{array}{l}\text { Houve redução modesta do risco de } \\
\text { CCR aos } 15 \text { anos. }\end{array}$ & 1 \\
\hline
\end{tabular}

Legenda: $\mathrm{AAS}=$ aspirina; $\mathrm{CCR}$ = carcinoma colorretal; RRA = redução do risco absoluto.

idade igual ou superior a 45 anos, saudáveis, que integraram um ECA (Women's Health Study), cujo objetivo foi determinar o efeito de 100mg de AAS, em dias alternados, no CCR após 10 anos de tratamento.

O presente estudo original é um follow-up desse ECA e avaliou o risco de CCR após 15 anos de tratamento, demonstrando redução modesta desse risco (Redução do Risco Absoluto (RRA)=0,14\%; IC95\% [0,02-0,59\%]). Por outro lado, a AAS mostrou aumentar o risco de HGI em todas as mulheres (RRA $=0,75 \%$; IC95\% $[0,50$ $1,00 \%])$.

Considerando o benefício absoluto da AAS no CCR e o risco hemorrágico concluiu-se que a AAS pode não ser uma opção adequada na maioria das mulheres. O risco hemorrágico é maior nas mulheres com 65 ou mais anos, mas os benefícios no risco de CCR foram também superiores nestas, podendo ponderar-se a utilização da AAS neste grupo etário.

Trata-se de um estudo de elevada qualidade (tamanho adequado e follow-up superior a $80 \%$ ). Embora apresente espectro de participantes que pode não ser representativo da população geral (mulheres saudáveis e profissionais de saúde), poderá atribuir-se NE 1 pela escala SORT.

\section{CONCLUSÕES}

Globalmente, os estudos parecem mostrar que a AAS, na dose igual ou superior a $300 \mathrm{mg} / \mathrm{dia}$, adminis- 
trada durante cinco ou mais anos, poderá reduzir a incidência de CCR, com efeito mais pronunciado para além dos dez anos após o início da toma. Doses inferiores, menos frequentes e com duração de tratamento inferior parecem ser menos efetivas. Contudo, a dose igual ou superior a $75 \mathrm{mg} /$ dia poderá ser eventualmente eficaz a longo prazo, como demonstrado por Rothwell e colaboradores (NE 1). ${ }^{3}$

Desta forma, e apesar de se constatar que muitos estudos têm sido realizados em relação a esta temática, continua-se a perceber que a evidência é limitada.

Parece necessário realizar mais ECAs em grandes populações de médio risco para CCR. É também fundamental existir maior clareza na idade a partir da qual existe benefício oncológico para a administração da AAS.Mais estudos deverão focar-se na incidência e mortalidade por CCR, enquanto outcomes primários.

Permanece muita inconsistência de resultados nomeadamente entre ECAs e estudos observacionais de qualidade limitada. Estudos futuros deverão garantir homogeneidade nas doses de AAS e duração do tratamento, ou seja, é necessário diminuir a heterogeneidade dos estudos e assegurar follow-up a longo prazo que permita esclarecer o potencial efeito da AAS.

Será também necessário que a investigação futura determine o impacto na redução do risco relativo de CCR com a administração da AAS, assim como o número necessário a tratar de indivíduos para reduzir a incidência e mortalidade por CCR.

Um achado interessante, demonstrado por Cesare Hassan e colaboradores, ${ }^{10}$ é o potencial efeito de «quimioprevenção» da AAS na redução adicional da incidência de CCR, quando combinada com a CT. A redução particularmente significativa da incidência e mortalidade por carcinoma do cólon proximal com a AAS, demonstrada por Rothwell e colaboradores, ${ }^{3}$ poderá ser também um achado promissor ao colmatar a potencial dificuldade da CT na deteção do carcinoma do cólon proximal.

Embora os efeitos colaterais da AAS sejam objetivos secundários desta revisão, parece essencial ter em consideração que estes podem surgir, nomeadamente as complicações hemorrágicas. Estas deverão ser também estudadas em ECAs futuros no sentido de esclarecer a dose e a duração mínima que permita ao indivíduo ter benefício oncológico máximo com o mínimo risco he- morrágico. Para isto, é também necessário ter em consideração as comorbilidades aquando da decisão de administrar ou não a AAS.

De acordo com o corpo de evidências analisado nesta revisão conclui-se que a AAS, administrada de forma regular, poderá ser efetiva na redução da incidência do CCR na população estudada (população adulta de risco médio para CCR), com eventual impacto na mortalidade por CCR e com uma força de recomendação B (SORT B). De futuro, será necessário avaliar o impacto na redução do risco relativo de CCR e o número necessário a tratar de indivíduos para reduzir a incidência e mortalidade por CCR, assim como definir a dose necessária de AAS a administrar.

\section{REFERÊNCIAS BIBLIOGRÁFICAS}

1. Ferlay J, Steliarova-Foucher E, Lortet-Tieulent J, Rosso S, Coebergh JW, Comber $\mathrm{H}$, et al. Cancer incidence and mortality patterns in Europe: estimates for 40 countries in 2012. Eur J Cancer. 2013;49(6):1374-403.

2. Rostom A, Dube C, Lewin G. Use of aspirin and NSAIDs to prevent colorectal cancer [Internet]. Rockville (MD): Agency for Healthcare Research and Quality; 2007.Available from: https://www.ncbi.nlm.nih.gov/ books/NBK33464/pdf/Bookshelf_NBK33464.pdf

3. Rothwell PM, Wilson M, Elwin CE, Norrving B, Algra A, Warlow CP, et al. Long-term effect of aspirin on colorectal cancer incidence and mortality: 20-year follow-up of five randomised trials. Lancet. 2010;376 (9754):1741-50.

4. Flossmann E, Rothwell PM. Effect of aspirin on long-term risk of colorectal cancer: consistent evidence from randomised and observational studies. Lancet. 2007;369(9573):1603-13.

5. Van Kruijsdijk RC, Visseren FL, Ridker PM, Dorresteijn JA, Buring JE, van der Graaf $Y$, et al. Individualized prediction of alternate-day aspirin treatment effects on the combined risk of cancer, cardiovascular disease and gastro-intestinal bleeding in healthy women. Heart. 2015;101 (5):369-76.

6. Ebell MH, Siwek J, Weiss BD, Woolf SH, Susman J, Ewigman B, et al. Strength of Recommendation Taxonomy (SORT): a patient-centered approach to grading evidence in the medical literature. Am Fam Physician. 2004;69(3):548-56.

7. U.S. Preventive Services Task Force. Routine aspirin or nonsteroidal antiinflammatory drugs for the primary prevention of colorectal cancer: U.S. Preventive Services Task Force recommendation statement. Ann Intern Med. 2007;146(5):361-4.

8. Sutcliffe P, Connock M, Gurung T, Freeman K, Johnson S, Kandala NB, et al. Aspirin for prophylactic use in the primary prevention of cardiovascular disease and cancer: a systematic review and overview of reviews. Health Technol Assess. 2013;17(43):1-253.

9. Dubé C, Rostom A, Lewin G, Tsertsvadze A, Barrowman N, Code C, et al. The use of aspirin for primary prevention of colorectal cancer: a systematic review prepared for the U.S. Preventive Services Task Force. Ann Intern Med. 2007;146(5):365-75. 
10. Hassan C, Rex DK, Cooper GS, Zullo A, Launois R, Benamouzig R. Primary prevention of colorectal cancer with low-dose aspirin in combination with endoscopy: a cost-effectiveness analysis. Gut. 2012;61(8): 1172-9.

\section{ENDEREÇO PARA CORRESPONDÊNCIA}

Sílvia Comonero Martins

E-mail: silvia_colmonero@hotmail.com

https://orcid.org/0000-0002-1600-8571

Recebido em 17-07-2017
Aceite para publicação em 10-09-2018

\section{CONFLITO DE INTERESSES}

As autores declaram não ter conflitos de interesses.

\section{ABSTRACT \\ WHAT IS THE ROLE OF ASPIRIN IN PRIMARY PREVENTION OF COLORECTAL CANCER IN MEDIUM-RISK INDIVIDUALS? AN EVIDENCE-BASED REVIEW}

Introduction: Colorectal cancer (CRC) is the second leading cause of cancer-related mortality in developed countries. Primary prevention is bases on lifestyle changes, and chemoprophylaxis with aspirin ${ }^{\circledR}$ is currently under investigation. Some studies have shown benefit of aspirin ${ }^{\circledR}$ in the prevention of CRC.

Objective: To determine the evidence the effect of aspirin ${ }^{\circledR}$ on CRC incidence and mortality in medium-risk adult subjects. Methods: Randomised controlled trials, meta-analyses, systematic reviews, evidence based guidelines, as well as Portuguese primary care journals and electronic databases, including MEDLINE and Cochrane Library, were searched. Searches were limited to publications between 1/4/2006 and 1/4/2016, in Portuguese, English or Spanish and used the MeSH terms: 'Aspirin','Primary prevention', and 'Colorectal neoplasms'. The Strength of Recommendation Taxonomy (SORT) of the American Academy of Family Physicians was used to rate the strength of a recommendation and the levels of evidence.

Results: Seventy-five papers were obtained, amongst which seven met the inclusion criteria: one evidence-based guideline, one meta-analysis, three systematic reviews, one cohort study, and one original study. The guideline does not recommend aspirin ${ }^{\circledR}$ for CRC prevention in medium risk individuals (SORT B). Overall, the studies seem to show that aspirin ${ }^{\circledR}$, when administrated for $\geq 5$ years at a dose $\geq 300 \mathrm{mg} /$ day, may reduce the incidence of $C R C$, with a more pronounced effect 10 years after starting treatment. Lower doses given less frequently or shorter treatment duration appear to be less effective.

Conclusions: When regularly administrated, aspirin ${ }^{\circledR}$ may reduce the incidence of $C R C$, with a possible impact on CRC mortality (SORT B). However, the use of aspirin ${ }^{\circledR}$ should be recommended with caution because of the increased incidence of adverse, such as gastrointestinal bleeding. Further RCTs are needed to clarify which dose and duration provide the maximum cancer benefit and minimal haemorrhagic risk.

Keywords: Aspirin; Primary prevention; Colorectal neoplasms. 